\title{
Support Service Use and Interest in Support Services among Distressed Family Caregivers of Lung Cancer Patients
}

\author{
Catherine E. Mosher, Ph.D. ${ }^{1}$, Victoria L. Champion, Ph.D., R.N., F.A.A.N. ${ }^{2}$, Nasser Hanna, \\ M.D. ${ }^{3}$, Shadia I. Jalal, M.D. ${ }^{3}$, Achilles J. Fakiris, M.D. ${ }^{4}$, Thomas J. Birdas, M.D. ${ }^{5}$, Ikenna C. \\ Okereke, M.D. ${ }^{5}$, Kenneth A. Kesler, M.D. ${ }^{5}$, Lawrence H. Einhorn, M.D. ${ }^{3}$, Barbara A. Given, \\ Ph.D., R.N., F.A.A.N. ${ }^{6}$, Patrick O. Monahan, Ph.D. ${ }^{7}$, and Jamie S. Ostroff, Ph.D. ${ }^{8}$ \\ ${ }^{1}$ Department of Psychology, Indiana University-Purdue University Indianapolis, Indianapolis, IN \\ ${ }^{2}$ Indiana University School of Nursing, Indianapolis, IN \\ ${ }^{3}$ Department of Medicine, Indiana University School of Medicine, Indianapolis, IN \\ ${ }^{4}$ Department of Radiation Oncology, University of North Carolina at Chapel Hill, Chapel Hill, NC \\ ${ }^{5}$ Department of Surgery, Indiana University School of Medicine, Indianapolis, IN \\ ${ }^{6}$ College of Nursing, Michigan State University, East Lansing, MI \\ ${ }^{7}$ Department of Biostatistics, Indiana University School of Medicine, Indianapolis, IN \\ ${ }^{8}$ Department of Psychiatry and Behavioral Sciences, Memorial Sloan-Kettering Cancer Center, \\ New York, NY
}

\section{Abstract}

Objective-This study examined support service use and interest in support services among distressed family caregivers of patients recently entering a comprehensive cancer care facility.

\begin{abstract}
Methods-Primary family caregivers of lung cancer patients $(N=83)$ were recruited from three medical centers within 12 weeks of the patient's new visit to the oncology clinic. All family caregivers were screened for psychological distress and those reporting significant anxiety or depressive symptoms were eligible for this study. Caregivers completed a baseline assessment of support service use (i.e., use of mental health services and complementary and alternative medicine $[\mathrm{CAM}]$ ) and interest in support services. Support service use was also assessed three months later.
\end{abstract}

Results-Although all caregivers reported clinically meaningful distress, only $26 \%$ used mental health and 39\% used CAM services during the 3-month study period. Patients' receipt of chemotherapy was positively associated with caregivers' mental health service use, whereas greater education and receiving assistance with caregiving tasks were associated with CAM use. Forty percent of caregivers who did not use CAM at baseline were interested in CAM. In addition, $29 \%$ of caregivers who did not receive mental health services at baseline were interested in professional psychosocial support, and $29 \%$ of caregivers who did not receive staff assistance with practical needs at baseline were interested in this service.

Correspondence concerning this article should be addressed to Catherine E. Mosher, Ph.D., Department of Psychology, Indiana University-Purdue University Indianapolis, 402 North Blackford Street, LD 124, Indianapolis, IN 46202. Phone: 317-274-6769. Fax: 317-274-6756. cemosher@iupui.edu.

Authors' Disclosures of Potential Conflicts of Interest

The authors indicated no potential conflicts of interest. 
Conclusions-Findings suggest that distressed family caregivers of lung cancer patients underuse mental health services and that a sizable minority are interested in professional help with psychosocial and practical needs.

\section{Keywords}

lung cancer; oncology; family caregivers; mental health service use; complementary and alternative medicine; psychological distress

Lung cancer is one of the most common cancers affecting both men and women, with over 220,000 new cases in the United States expected in 2012 [1]. Most lung cancer patients $(85 \%)$ have regional or distant stage disease at diagnosis [1], contributing to their high rate $(80 \%)$ of multiple physical and psychological symptoms [2-4]. Family caregivers often provide informational, emotional, or financial support to ill relatives or friends as well as assistance with medical or personal care [5-6]. Given the extent of family caregiving responsibilities, it is not surprising that up to $50 \%$ of cancer patients' family caregivers report significant distress [7-12].

Lung cancer may be especially distressing for family caregivers because of its high physical symptom burden [13], which may increase caregiving demands, and possible attributions of blame or stigma associated with the patient's tobacco use [14]. Caregivers of lung cancer patients have reported difficulty with caregiving tasks such as providing emotional support, managing behavior problems, and monitoring symptoms [15]. Spousal caregivers of lung cancer patients have also reported difficulty discussing the patient's prognosis, symptom management, persistent tobacco use, and their own emotions [16]. One third of spousal caregivers of lung cancer patients have been found to experience clinically elevated anxiety or depressive symptoms [10-11]. Another study of lung cancer patients' caregivers that included relatives (e.g., spouses, adult children) and friends found that about $40 \%$ reported high levels of strain on scales assessing depressive symptoms, exhaustion, and emotional well-being [7].

Although a growing literature has documented the psychosocial impact of lung and other cancers on family caregivers [17-19], their rates of psychosocial support service use are typically low. In a nationally representative survey of primary informal caregivers of chronically disabled community-dwelling older adults, including those with cancer, less than $5 \%$ of caregivers reported participating in a caregiver support group or using respite services [20]. Another study found that fewer than half (46\%) of advanced cancer patients' caregivers with a psychiatric diagnosis sought mental health care [21]. Little is known about caregivers' complementary and alternative medicine (CAM) use and interest in CAM and other support services.

What factors might explain the observation that despite caregivers of cancer patients reporting high rates of distress [7-8, 12], they tend to report low rates of mental health service use [21]? Knowledge about caregivers' interest in a range of support services and a greater understanding of factors that are predictive of support service use is essential for guiding the development of interventions that are acceptable and readily sustainable in clinical practice.

To address these gaps in the literature, the present study examined use of mental health services and CAM and interest in support services among distressed family caregivers of lung cancer patients. Distress was defined as meeting the clinical cutoff for significant anxiety or depressive symptoms on a standardized and widely used self-report measure. Caregivers' use of mental health services and CAM was assessed within 12 weeks of the 
patient's initial visit to the oncology clinic and three months later to determine uptake of services during the early phase of cancer care at comprehensive medical centers. We focused on the early phase of care because it represents an initial period of heightened distress and immersion into caregiving responsibilities [10]. Family caregivers who report heightened distress during the initial phase of treatment are likely to benefit from early identification and encouragement to access available support services [19].

Andersen's behavioral model of healthcare use [22] was the organizing framework for the current study. This widely supported model has substantial relevance to understanding use of mental health services and CAM [23]. The model posits that healthcare use is influenced by three factors: 1) predisposing factors such as gender, age, race, marital status, education, and occupation; 2) enabling or access-related factors such as income, health insurance, and transportation; and 3) need for services as indicated by psychiatric symptoms and perceptions of functional capacity. An example of these perceptions is coping self-efficacy (e.g., confidence in one's ability to solve problems or stop unpleasant thoughts and emotions) [24]. The present study assessed predisposing, enabling, and need variables as potential correlates of mental health service use, CAM use, and interest in support services.

The aims of this study are: (1) to examine distressed caregivers' use of mental health services (e.g., psychotherapy, medication) and CAM (e.g., yoga, meditation, massage) during the initial period of care at an oncology clinic; (2) to determine caregivers' interest in various support services (e.g., talking to a staff member about one's feelings, support group, CAM, professional help with practical needs); and (3) to examine the extent to which predisposing factors (e.g., age, gender), enabling factors (e.g., income, transportation), and need variables (i.e., anxiety and depressive symptoms and coping self-efficacy) are associated with caregivers' use of mental health services and CAM and interest in mental health services (i.e., talking to a staff member about one's feelings, support group), CAM, and professional help with practical needs. The extent to which patient medical factors (e.g., cancer stage, cancer treatments) were correlated with these outcomes also was explored.

Based on Andersen's model of healthcare use [22] and prior research [25-29], the following correlates of mental health service use, CAM use, and interest in mental health services and CAM were hypothesized:

1. Predisposing factors would include younger age, female gender, unmarried status, greater education, and less caregiving responsibility (i.e., not caring for multiple family members).

2. Enabling factors would include greater income, fewer transportation difficulties, and the receipt of caregiving assistance.

3. Need variables would include greater anxiety and depressive symptoms and lower levels of coping self-efficacy.

We also hypothesized that lower levels of income and education, greater transportation difficulties, and greater caregiving responsibility would be associated with greater interest in professional help with practical needs.

\section{Methods}

\section{Participants and Procedures}

Family caregivers of lung cancer patients were recruited by contacting eligible patients from the oncology clinics at Memorial Sloan-Kettering Cancer Center (MSKCC; New York, NY), Indiana University Simon Cancer Center (IUSCC; Indianapolis, IN), and the Roudebush VA Medical Center (Indianapolis, IN). All study procedures were approved by the institutional review boards at each site. Eligible lung cancer patients were English speakers who were 
within 12 weeks of their initial visit to the oncology clinic. Eligible patients did not have lung cancer recurrence. Review of medical records and consultation with oncologists confirmed patient eligibility. A research assistant approached the patient during a clinic visit to describe the study. Interested patients identified their primary family caregiver (i.e., the person who provides the majority of their unpaid, informal care) and provided written consent to contact their caregiver and collect cancer-related information from their medical record. Initial inclusion requirements for caregivers were: (1) English fluency; and (2) at least 18 years of age. All caregivers were screened for clinically significant distress by a trained research assistant and eligible caregivers reported scores exceeding the clinical cutoff ( $\geq 8)$ on the Anxiety or Depression subscales of the Hospital Anxiety and Depression Scale (HADS) [30-31]. A research assistant completed the eligibility screening and informed consent process in clinic or via telephone. All caregivers who completed the distress screening tool received a brochure outlining psychosocial support services available at the study site. Each of the three study sites offered comprehensive mental health services (e.g., psychiatric, psychological, and social work services).

Baseline telephone assessments included questions regarding demographic and medical information, support service use (i.e., receipt of mental health services, CAM, and staff assistance with practical needs), interest in support services, and coping self-efficacy. Use of mental health and CAM services also was assessed via telephone three months later. Caregivers received $\$ 25$ for the baseline assessment and \$25 for the follow-up assessment.

\section{Measures}

Medical information-Information regarding the patient's type and stage of lung cancer, time since diagnosis, and cancer treatment (i.e., chemotherapy, radiation, or surgery) was collected from medical records. At baseline, caregivers also reported whether the patient had received chemotherapy, radiation, or surgery.

Support service use-At baseline, caregivers reported whether they were currently receiving mental health services. At follow-up, caregivers reported whether they had received mental health services since the baseline assessment. These services included psychotherapy/counseling, psychotropic medications, support groups, or visits to clergy. If they were receiving services, they were asked to identify the type of service received. In addition, caregivers indicated whether they were currently receiving CAM (i.e., yoga, meditation, hypnosis, massage, or another service) at baseline. At follow-up, caregivers reported whether they had received CAM since the baseline assessment. Staff assistance with practical needs such as transportation and finances was only assessed at baseline.

Interest in support services-Caregivers' interest in support services was assessed with a series of yes or no questions regarding six services available through the cancer center or in the community. Support services included psychosocial services (i.e., talking to a staff member about your feelings and how you are coping with the illness, support group, family counseling, couples counseling), CAM (i.e., yoga, meditation, hypnosis, massage, or another service), and professional help with practical needs (e.g., transportation or finances).

Additional yes or no questions assessed whether caregivers had access to email, used the internet on a weekly basis, and preferred communicating with a counselor via telephone or the internet, respectively.

\section{Predisposing variables}

1. Demographics. Caregivers reported the following demographic information: age, gender, ethnicity, marital status, education, and employment status. 
2. Caregiver-patient relationship. Questions assessed whether the caregiver and patient shared the same household and the nature of the relationship between the caregiver and patient (e.g., spouse/partner, adult child).

3. Caregiving responsibility. Caregivers reported whether they cared for young children or another family member with an illness or disability.

\section{Enabling variables}

1. Health insurance status and income. Caregivers reported whether they received health insurance and their income level.

2. Caregiving assistance. Caregivers indicated whether they received caregiving assistance from paid or unpaid helpers within or outside the home [32].

3. Transportation difficulties. Difficulty traveling to appointments for medical or supportive services was assessed on a 4-point scale from 1 (not at all difficult) to 4 (extremely difficult) [33].

\section{Need variables}

1. Anxiety and depressive symptoms. The 14-item Hospital Anxiety and Depression Scale [30] assessed caregivers' symptoms of anxiety and depression during the past week at enrollment. The HADS has been found to be valid and reliable [31]. Coefficient alphas for Anxiety and Depression subscales in the present research were .69 and .76 , respectively.

2. Coping self-efficacy. The Coping Self-Efficacy Scale [24] assessed caregivers' confidence in their ability to use problem-focused coping, stop unpleasant emotions and thoughts, and receive support from friends and family. Each of the 13 items was rated on an 11-point scale $(0=$ cannot do at all to $10=$ certain can do $)$. This scale has demonstrated reliability and predictive validity in studies of people coping with chronic illness [24]. Coefficient alpha for the present research was .92.

\section{Statistical Analyses}

Data were analyzed with SPSS statistical software (version 19.0; SPSS, Chicago, IL, USA). First, descriptive statistics were computed to characterize caregivers' demographic information, patients' medical variables, and caregivers' receipt of mental health services, CAM, and staff assistance with practical needs, interest in support services, and coping selfefficacy. Chi-square analyses and t-tests were used to examine whether demographic and medical factors and study variables differed between participants from two of the study sites (i.e., MSKCC and IUSCC). Next, logistic regression models estimated the unadjusted, bivariate effects of predisposing factors (i.e., caregiver demographics, caregiver-patient relationship status, and caregiving responsibility), enabling factors (i.e., income, caregiving assistance, and transportation), need variables (i.e., caregiver anxiety and depressive symptoms and coping self-efficacy), and patient medical factors (i.e., disease stage, cancer treatments, and time since diagnosis) on caregivers' mental health service use and CAM use at either study time point (i.e., baseline or 3-month follow-up) and interest in support services at baseline (i.e., interest in talking to a staff member about their feelings, support group, CAM, and professional help with practical needs). A second set of adjusted logistic regression models simultaneously estimated all significant bivariate effects. All reported $p$ values were 2 -sided and a value of $p<.05$ was considered statistically significant. 


\section{Results}

\section{Sample characteristics}

Of the 234 potentially eligible lung cancer patients who were approached regarding this study, 97\% $(n=227)$ identified a family caregiver. Most patients $(97 \%, n=221)$ allowed the research assistant to contact their caregiver. The majority of caregivers $(86 \%, n=189)$ agreed to complete the HADS to determine their eligibility status, $12 \%$ declined to participate, and $2 \%$ were unable to be reached via phone. Primary reasons for study refusal were time constraints, personal stress, and a desire to focus on the patient's needs. Half of caregivers $(50 \%, n=95$ of 189) met the clinical cutoff (score 28 ) on the Anxiety or Depression subscale of the HADS. Most eligible distressed caregivers $(95 \%, n=90)$ consented to participate in this study. Eighty-three caregivers (92\%) completed the baseline phone assessment, and 74 caregivers completed the follow-up phone assessment (82\% retention). Reasons for withdrawal during the study period included time constraints, personal illness, bereavement, and inability to reach the caregiver via phone.

Demographic and medical characteristics of the sample are shown in Table 1. Participants were, on average, 55 years old, married, female, Caucasian, and well-educated (mean $=15$ years of education). The median annual household income was over $\$ 50,000$ with a broad range. Over half of caregivers were working full or part-time and received paid or unpaid assistance with caregiving. Most caregivers were spouses/partners (65\%) or adult children (25\%) of the patient, and $25 \%$ were caring for two or more family members, including the patient. The majority of patients (60\%) were diagnosed with stage III or IV non-small cell lung cancer. At baseline, patients were, on average, 8 weeks from the lung cancer diagnosis. Five of the caregivers were bereaved at follow-up. Average coping self-efficacy was also relatively high $(M=83.76, S D=23.59)$ with some variability observed.

MSKCC and IUSCC participants were compared with regard to demographic and medical factors and study variables. MSKCC caregivers reported greater education, $t(74)=2.70, p$ $<.01$, and income, $t(70)=4.78, p<.001$, relative to IUSCC caregivers. MSKCC caregivers also were more likely to be caring for multiple family members than IUSCC caregivers, $\chi^{2}$ $(1, N=76)=6.06, p<.05$. IUSCC participants were more likely to be caring for a patient who had undergone radiation than MSKCC participants, $\chi^{2}(1, N=76)=4.28, p<.05$. Finally, MSKCC caregivers reported greater difficulty traveling to appointments, $t(74)=$ $4.03, p<.001$, and greater interest in professional help with practical needs (e.g., transportation, finances), $\chi^{2}(1, N=75)=9.31, p<.01$, than IUSCC caregivers. None of the other variables differed between the two study sites.

\section{Support Service Use}

At baseline, only $13 \%$ of these distressed caregivers (11/83) were receiving mental health services. These services included psychotherapy (5/11), psychotropic medication (6/11), and support groups (2/11). At follow-up, 19\% of caregivers (14/74) indicated that they had received mental health services during the 3-month study period. Of the 14 caregivers who accessed mental health services, 10 of them had not been receiving services at baseline. The most common mental health services were psychotropic medication (8/14) and counseling/ psychotherapy (6/14). Three caregivers had received support from a spiritual leader and one caregiver had attended a support group. A substantial minority of caregivers reported CAM use (e.g., yoga, meditation, massage) at baseline $(21 / 83,25 \%)$ and follow-up $(22 / 72,31 \%)$. Taken together, only $26 \%$ of participants with complete data (19/74) reported mental health service use and only $39 \%(28 / 72)$ reported CAM use at either study time point. In addition, only $4 \%$ of caregivers $(3 / 83)$ received assistance from a staff member with practical needs such as transportation and finances at baseline. 
Next, we examined the extent to which predisposing factors (i.e., caregiver demographics, caregiver-patient relationship status, and caregiving responsibility), enabling factors (i.e., income, caregiving assistance, and transportation), need variables (i.e., caregiver anxiety and depressive symptoms and coping self-efficacy), and patient medical factors were associated with use of mental health services and CAM at either study time point. In bivariate analyses, mental health service use was associated with higher levels of anxiety at screening and the patient's receipt of chemotherapy at baseline (see Table 2). Multivariate logistic regression analyses revealed that these two variables correctly classified $78 \%$ of the sample with regard to mental health service use; however, only the patient's receipt of chemotherapy uniquely predicted mental health service use. In bivariate analyses, CAM use was associated with greater education, being unmarried, receiving paid or unpaid assistance with caregiving tasks, and higher levels of anxiety at screening (see Table 2). In the multivariate logistic regression model, these four variables correctly classified $75 \%$ of the sample with regard to CAM use, but only greater education and receiving assistance with caregiving tasks were significant unique predictors of this outcome.

\section{Interest in Support Services}

Caregivers' interest in psychosocial and instrumental support services was assessed. Forty percent of caregivers (25/62) who did not use CAM at baseline were interested in these services, and $29 \%$ of caregivers (21/72) who did not receive mental health services at baseline were interested in attending a support group or talking to a staff member about their feelings, respectively. Fewer caregivers expressed interest in couples counseling $(7 / 46,15 \%)$ or family counseling $(14 / 72,19 \%)$. When asked about their preferred mode of delivery for mental health services, more caregivers were interested in having a counselor be available via telephone $(57 / 72,79 \%)$ than via the internet $(34 / 72,47 \%)$, although the vast majority had email access $(67 / 72,93 \%)$ and used the internet on a weekly basis $(64 / 72,89 \%)$. Of caregivers who did not receive staff assistance with practical needs (e.g., transportation, finances) at baseline, 29\% (23/79) were interested in this assistance.

The extent to which predisposing factors (i.e., caregiver demographics, caregiver-patient relationship status, and caregiving responsibility), enabling factors (i.e., income, caregiving assistance, and transportation), need variables (i.e., caregiver anxiety and depressive symptoms and coping self-efficacy), and patient medical factors were correlated with interest in specific support services among caregivers who did not access these services at baseline was examined. Results are shown in Table 2. In bivariate analyses, interest in talking to a staff member about one's feelings was associated with greater years of education, whereas interest in attending a support group was associated with late-stage disease. Interest in CAM was correlated with being a caregiver who was not the spouse or partner of the patient. Interest in professional help with practical needs was correlated with caring for two or more family members, greater difficulty traveling to appointments, lower coping self-efficacy, and shorter time since diagnosis. In the multivariate logistic regression model, these four variables correctly classified $76 \%$ of caregivers with regard to interest in help with practical needs; however, only greater difficulty traveling to appointments, lower coping self-efficacy and shorter time since diagnosis were unique predictors of this outcome. Correlates of interest in family and couples counseling were not examined due to the small number of participants endorsing those items.

\section{Discussion}

The present study provides initial data regarding mental health and CAM service use and interest in support services among distressed family caregivers of lung cancer patients. Half of family caregivers who were screened for study eligibility showed clinically significant anxiety or depressive symptoms, which is higher than that reported in prior research with 
lung cancer patients' family caregivers [7, 10-11]. Variability in caregiver and patient characteristics (e.g., age, type of relationship), the timing of assessments, and the measurement of distress may account for differential rates of caregiver distress across studies. Although all caregivers reported clinically meaningful distress and most had health insurance, only $26 \%$ used mental health services (e.g., psychotherapy, medication) and 39\% used CAM services (e.g., yoga, meditation, massage) during the first several months shortly after the patient's initial visit to the oncology clinic. During this early phase of cancer care at comprehensive medical centers that offered numerous support services, the majority of distressed caregivers reported nonuse of available mental health and CAM services.

Similarly, a cross-sectional study found that less than half of cancer patients' caregivers with a psychiatric disorder received mental health care [21].

Based on Andersen's model of healthcare use [22] and prior research with population-based samples [25-29], we had hypothesized that predisposing factors (e.g., younger age, female gender), enabling factors (e.g., greater income, fewer transportation difficulties), and need variables (i.e., greater anxiety and depressive symptoms and lower levels of coping selfefficacy) would be associated with use of mental health services and CAM. Contrary to hypotheses, the patients' receipt of chemotherapy was the only unique predictor of caregivers' mental health service use. Common stressors associated with chemotherapy (e.g., serious side effects, poor prognosis) may increase family members' distress and caregiving demands, which may, in turn, prompt some caregivers to seek professional support. As hypothesized, CAM use was associated with greater education and the receipt of caregiving assistance. Further research with larger and more socioeconomically diverse samples of cancer patients' caregivers is needed to determine predisposing, enabling, and need variables associated with use of mental health services and CAM.

Caregivers' interest in specific support services also was assessed. Forty percent of caregivers who did not use CAM at baseline were interested in CAM, and 29\% of caregivers who did not receive mental health services at baseline were interested in attending a support group or talking to a staff member about their feelings, respectively. In addition, 29\% of caregivers who were not receiving professional help with practical needs (e.g., transportation, finances) at baseline were interested in this help. Fewer caregivers were interested in couples or family counseling, consistent with prior research with cancer patients' caregivers [34]. The current results parallel caregivers' use of support services and indicate that the majority of distressed caregivers are not interested in using traditional mental health services for help in dealing with emotional concerns. However, the majority of caregivers who did not use mental health services at baseline (79\%) expressed interest in having a counselor be available via telephone if they were to have an emotional concern. Although the vast majority of caregivers had access to the internet, fewer caregivers were interested in receiving counseling via the internet (47\%). Internet use has been found to be a more effortful process for older age groups [35]; thus, this sample of primarily middle-aged and older adults appears to have more interest in phone-based than internet-based services.

When examining correlates of interest in mental health services and CAM, few predictions based on Andersen's model [22] were supported. Only one predisposing factor (i.e., higher levels of education) was correlated with greater interest in talking to a staff member about one's feelings. None of the variables from Andersen's model were correlated with caregivers' interest in support groups. However, late-stage disease was correlated with interest in support groups, suggesting that peer support may be valued when facing a poor prognosis. Finally, interest in CAM was associated with being a caregiver who was not the spouse or partner of the patient, a factor related to younger age. 
Limitations of this study and directions for future research warrant discussion. First, the sample primarily consisted of Caucasian, middle class women who were either spousal or adult child caregivers. Whether findings would generalize to other groups (e.g., ethnic minority group members, individuals of lower socioeconomic status) requires study. In addition, the relatively small sample size reduced the statistical power for detecting significant correlates of study outcomes. Further research with larger samples is needed to replicate and extend the current findings. Although support service use was assessed longitudinally, a third study limitation is the cross-sectional assessment of interest in support services and other caregiver characteristics. Future research may examine changes in caregivers' support service use and interest in services over the course of the illness. Such research may examine other potential predictors of caregivers' support service use and interest in services such as stigma associated with lung cancer or mental health service use, illness-related attributions (e.g., blame), and health risk behaviors (e.g., smoking, alcohol use).

The current findings have important implications for future research and clinical care. First, findings highlight high rates of significant distress and underuse of support services among family caregivers of lung cancer patients. In addition, a minority of distressed caregivers were interested in support services, and interest in specific services varied according to patient medical factors (e.g., disease stage) and caregiver characteristics (e.g., level of education). Research is needed to further identify subgroups of caregivers who are most receptive to support services as well as attitudinal and systemic barriers to support service use. In addition, research is required to develop and evaluate interventions that are tailored to the needs and preferences of lung cancer patients' caregivers. Our results suggest that caregivers may be most receptive to telephone-based interventions. Multidisciplinary efforts are needed to address caregivers' practical concerns and psychosocial support needs. Given the complex psychosocial issues related to the etiology of lung cancer and its morbidity, determining how best to meet caregivers' needs is an important goal for future research and clinical practice.

\section{Acknowledgments}

This study was supported by Grant No. R03CA139862 from the NCI. CM was supported by F32CA130600 from the NCI and KL2 RR025760 (A. Shekhar, PI) from the NCRR. The authors would like to thank the study participants, the thoracic oncology teams at Memorial Sloan-Kettering Cancer Center, Indiana University Simon Cancer Center, and the Roudebush VA Medical Center, Scarlett Ho, Aigul Amankeldi, Shannon M. Christy, and undergraduate students for their assistance.

\section{References}

1. American Cancer Society. Cancer facts and figures-2012. Atlanta, GA: Author; 2012.

2. Hopwood P, Stephens RJ. Symptoms at presentation for treatment in patients with lung cancer: implications for the evaluation of palliative treatment. The Medical Research Council (MRC) Lung Cancer Working Party. Br J Cancer. 1995; 71:633-636. [PubMed: 7533520]

3. Rolke HB, Bakke PS, Gallefoss F. Health related quality of life, mood disorders and coping abilities in an unselected sample of patients with primary lung cancer. Respir Med. 2008; 102:1460-1467. [PubMed: 18590954]

4. Kurtz ME, Kurtz JC, Stommel M, Given CW, Given B. Predictors of depressive symptomatology of geriatric patients with lung cancer-a longitudinal analysis. Psychooncology. 2002; 11:12-22. [PubMed: 11835589]

5. Nijboer C, Tempelaar R, Sanderman R, Triemstra M, Spruijt RJ, van den Bos GA. Cancer and caregiving: the impact on the caregiver's health. Psychooncology. 1998; 7:3-13. [PubMed: 9516646] 
6. Stajduhar K, Funk L, Toye C, Grande G, Aoun S, Todd C. Part 1: Home-based family caregiving at the end of life: a comprehensive review of published quantitative research (1998-2008). Palliat Med. 2010; 24:573-593. [PubMed: 20562171]

7. Östlund U, Wennman-Larsen A, Persson C, Gustavsson P, Wengström Y. Mental health in significant others of patients dying from lung cancer. Psychooncology. 2010; 19:29-37. [PubMed: 19253315]

8. Braun M, Mikulincer M, Rydall A, Walsh A, Rodin G. Hidden morbidity in cancer: Spouse caregivers. J Clin Oncol. 2007; 25:4829-4834. [PubMed: 17947732]

9. Siegel K, Karus DG, Raveis VH, Christ GH, Mesagno FP. Depressive distress among the spouses of terminally ill cancer patients. Cancer Pract. 1996; 4:25-30. [PubMed: 8788767]

10. Carmack Taylor CL, Badr H, Lee JH, Fossella F, Pisters K, Gritz ER, et al. Lung cancer patients and their spouses: psychological and relationship functioning within 1 month of treatment initiation. Ann Behav Med. 2008; 36:129-140. [PubMed: 18797978]

11. Kim Y, Duberstein PR, Sörensen S, Larson MR. Levels of depressive symptoms in spouses of people with lung cancer: Effects of personality, social support, and caregiving burden. Psychosomatics. 2005; 46:123-130. [PubMed: 15774950]

12. Grunfeld E, Coyle D, Whelan T, Clinch J, Reyno L, Earle CC, et al. Family caregiver burden: results of a longitudinal study of breast cancer patients and their principal caregivers. Can Med Assoc J. 2004; 170:1795-1801. [PubMed: 15184333]

13. Spiro SG, Douse J, Read C, Janes S. Complications of lung cancer treatment. Semin Respir Crit Care Med. 2008; 29:302-317. [PubMed: 18506668]

14. Lobchuk MM, Murdoch T, McClement SE, McPherson C. A dyadic affair: who is to blame for causing and controlling the patient's lung cancer? Cancer Nurs. 2008; 31:435-443. [PubMed: 18987510]

15. Bakas T, Lewis RR, Parsons JE. Caregiving tasks among family caregivers of patients with lung cancer. Oncol Nurs Forum. 2001; 28:847-854. [PubMed: 11421144]

16. Badr H, Carmack Taylor CL. Social constraints and spousal communication in lung cancer. Psychooncology. 2006; 15:673-683. [PubMed: 16287210]

17. Ellis J. The impact of lung cancer on patients and carers. Chron Respir Dis. 2012; 9:39-47. [PubMed: 22308553]

18. Kim Y, Given BA. Quality of life of family caregivers of cancer survivors: across the trajectory of the illness. Cancer. 2008; 112:2556-2568. [PubMed: 18428199]

19. Northouse L, Williams AL, Given B, McCorkle R. Psychosocial care for family caregivers of patients with cancer. J Clin Oncol. 2012; 30:1227-1234. [PubMed: 22412124]

20. Wolff JL, Dy SM, Frick KD, Kasper JD. End-of-life care: Findings from a national survey of informal caregivers. Arch Intern Med. 2007; 167:40-46. [PubMed: 17210876]

21. Vanderwerker LC, Laff RE, Kadan-Lottick NS, McColl S, Prigerson HG. Psychiatric disorders and mental health service use among caregivers of advanced cancer patients. J Clin Oncol. 2005; 23:6899-6907. [PubMed: 16129849]

22. Andersen RM. Revisiting the behavioral model and access to medical care: does it matter? J Health Soc Behav. 1995; 36:1-10. [PubMed: 7738325]

23. Bruce ML, Wells KB, Miranda J, Lewis L, Gonzalez JL. Barriers to reducing burden of affective disorders. Ment Health Serv Res. 2002; 4:187-197. [PubMed: 12558003]

24. Chesney MA, Neilands TB, Chambers DB, Taylor JM, Folkman S. A validity and reliability study of the coping self-efficacy scale. Br J Health Psychol. 2006; 11:421-437. [PubMed: 16870053]

25. Wang PS, Lane M, Olfson M, Pincus HA, Wells KB, Kessler RC. Twelve-month use of mental health services in the United States: results from the National Comorbidity Survey Replication. Arch Gen Psychiatry. 2005; 62:629-640. [PubMed: 15939840]

26. Kessler RC, Demler O, Frank RG, Olfson M, Pincus HA, Walters EE, et al. Prevalence and treatment of mental disorders, 1990 to 2003. N Engl J Med. 2005; 352:2515-2523. [PubMed: 15958807]

27. Barnes PM, Bloom B, Nahin RL. Complementary and alternative medicine use among adults and children: United States: 2007. Natl Health Stat Report. 2008; 12:1-23. [PubMed: 19361005] 
28. Elhai JD, Ford JD. Correlates of mental health service use intensity in the National Comorbidity Survey and National Comorbidity Survey Replication. Psychiatr Serv. 2007; 58:1108-1115. [PubMed: 17664523]

29. Swartz MS, Wagner HR, Swanson JW, Burns BJ, George LK, Padgett DK. Administrative update: utilization of services. I. Comparing use of public and private mental health services: the enduring barriers of race and age. Community Ment Health J. 1998; 34:133-144. [PubMed: 9620158]

30. Zigmond AS, Snaith RP. The hospital anxiety and depression scale. Acta Psychiatr Scand. 1983; 67:361-370. [PubMed: 6880820]

31. Bjelland I, Dahl AA, Haug TT, Neckelmann D. The validity of the Hospital Anxiety and Depression Scale: An updated literature review. J Psychosom Res. 2002; 52:69-77. [PubMed: 11832252]

32. Toseland RW, McCallion P, Gerber T, Banks S. Predictors of health and human services use by persons with dementia and their family caregivers. Soc Sci Med. 2002; 55:1255-1266. [PubMed: 12365535]

33. Shapiro PJ, Coyne JC, Kruus LK, Palmer SC, Vaughn DJ, Malkowicz SB. Interest in services among prostate cancer patients receiving androgen deprivation therapy. Psychooncology. 2004; 13:512-525. [PubMed: 15295773]

34. Ostroff J, Ross S, Steinglass P, Ronis-Tobin V, Singh B. Interest in and barriers to participation in multiple family groups among head and neck cancer survivors and their primary family caregivers. Fam Process. 2004; 43:195-208. [PubMed: 15603503]

35. Ybarra M, Suman M. Reasons, assessments and actions taken: sex and age differences in uses of Internet health information. Health Educ Res. 2008; 23:512-521. [PubMed: 16880222] 
Table 1

Sample characteristics $(N=83)$

\begin{tabular}{|c|c|c|c|}
\hline Variable & $n(\%)$ & $M(S D)$ & Range \\
\hline Caregiver Sex-Female & $64(77 \%)$ & & \\
\hline \multicolumn{4}{|l|}{ Type of Relationship } \\
\hline Spouse/partner & $54(65 \%)$ & & \\
\hline Adult child & $21(25 \%)$ & & \\
\hline Sibling & $5(6 \%)$ & & \\
\hline Other relative & $3(4 \%)$ & & \\
\hline Caregiver lives with the patient & $62(75 \%)$ & & \\
\hline \multicolumn{4}{|l|}{ Caregiver Race/Ethnicity } \\
\hline Non-Hispanic White & $71(86 \%)$ & & \\
\hline African American/Black & $8(10 \%)$ & & \\
\hline Other & $4(5 \%)$ & & \\
\hline Caregiver Age (years) & & $55(11)$ & 29 to 80 \\
\hline \multicolumn{4}{|l|}{ Caregiver Marital Status } \\
\hline Married or marriage equivalent & $69(83 \%)$ & & \\
\hline Divorced & $7(8 \%)$ & & \\
\hline Single & $7(8 \%)$ & & \\
\hline Caregiver Annual Household Income (median) & & $>\$ 50,000$ & $<\$ 11,000$ to $>\$ 100,000$ \\
\hline Caregiver Education (years) & & $15(3)$ & 8 to 27 \\
\hline \multicolumn{4}{|l|}{ Caregiver Employment Status } \\
\hline Employed full or part time & $51(61 \%)$ & & \\
\hline Retired & $18(22 \%)$ & & \\
\hline Unemployed & $8(10 \%)$ & & \\
\hline Other & $6(7 \%)$ & & \\
\hline Caregiver has health insurance & $77(93 \%)$ & & \\
\hline Caregiver receives paid or unpaid assistance with caregiving & $45(54 \%)$ & & \\
\hline Caregiver cares for young children or another family member with an illness or disability & $21(25 \%)$ & & \\
\hline Weeks since Patient's Diagnosis at Baseline & & $8(8)$ & .14 to 64 \\
\hline \multicolumn{4}{|l|}{ Non-small Cell Lung Cancer Stage $(n=78)$} \\
\hline I & $16(21 \%)$ & & \\
\hline II & $10(13 \%)$ & & \\
\hline III & $20(26 \%)$ & & \\
\hline IV & $30(38 \%)$ & & \\
\hline Missing & $2(3 \%)$ & & \\
\hline \multicolumn{4}{|l|}{ Small Cell Lung Cancer Stage $(n=5)$} \\
\hline Limited & $2(40 \%)$ & & \\
\hline Extensive & $2(40 \%)$ & & \\
\hline Missing & $1(20 \%)$ & & \\
\hline \multicolumn{4}{|l|}{ Type of Treatment at Baseline } \\
\hline Surgery & $27(33 \%)$ & & \\
\hline
\end{tabular}




\begin{tabular}{|c|c|c|c|}
\hline Variable & $n(\%)$ & $M(S D)$ & Range \\
\hline Chemotherapy & $42(51 \%)$ & & \\
\hline Radiation & $30(36 \%)$ & & \\
\hline \multicolumn{4}{|l|}{ Treatment Center } \\
\hline \multicolumn{4}{|l|}{ Memorial Sloan-Kettering } \\
\hline Cancer Center (NY) & $43(52 \%)$ & & \\
\hline \multicolumn{4}{|l|}{ Indiana University Simon } \\
\hline Cancer Center (IN) & $33(40 \%)$ & & \\
\hline Roudebush VA Medical Center (IN) & $7(8 \%)$ & & \\
\hline
\end{tabular}




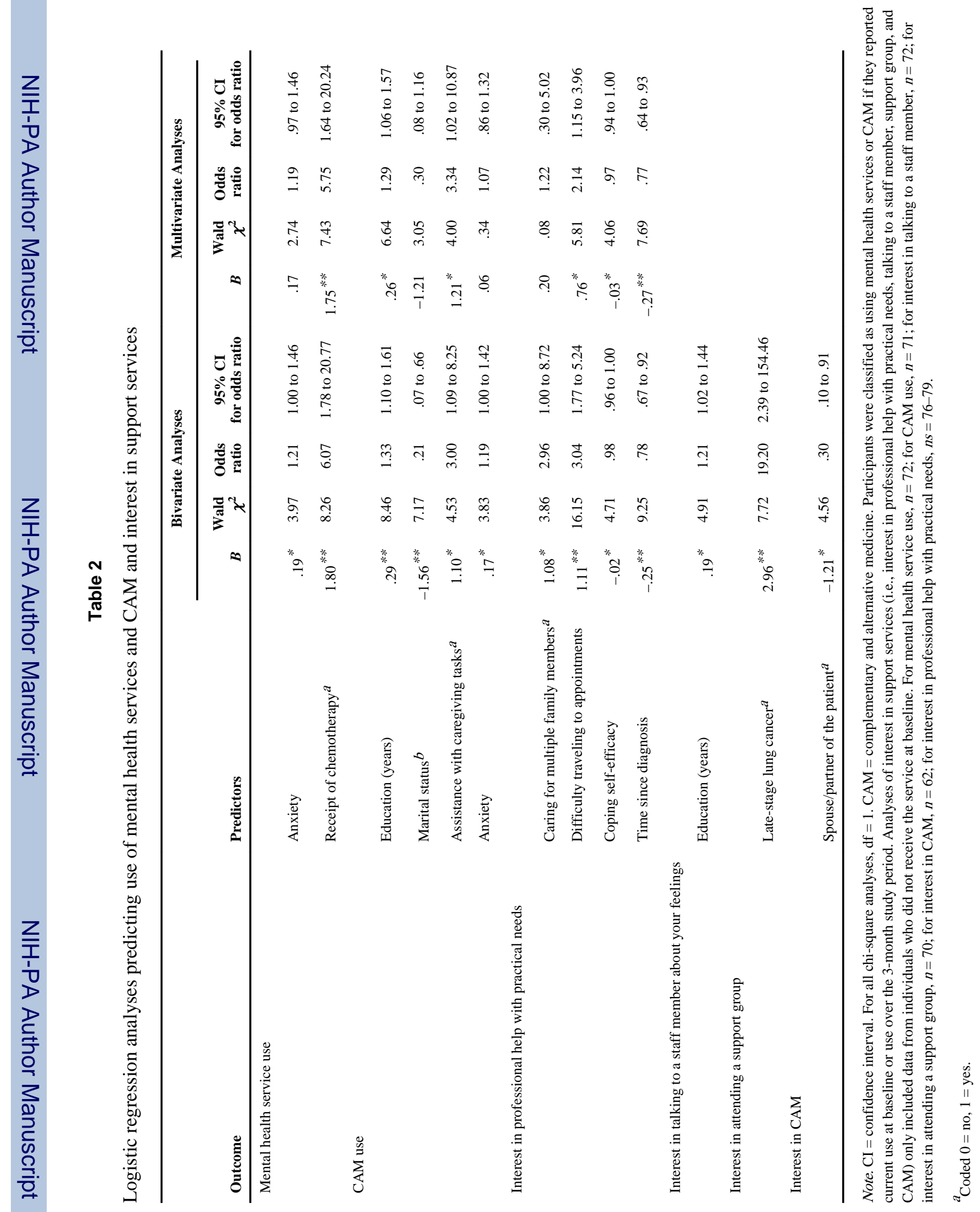


